\section{Evaluación de la tecnología empleada en la atención de la salud ${ }^{1}$}

1 Extractado de "La acción de la OPS en apoyo a la evaluación de las tecnologías en salud en América Latina y el Caribe", documento de trabajo del 16 de julio de 1997, originado por un grupo de trabajo coordinado por Alberto Infante, División de Desarrollo de Sistemas y Servicios de Salud, OPS.
La idea de evaluar el resultado de las intervenciones clínicas ha existido desde la antigüedad, pero el primer ensayo clínico registrado (para el tratamiento del escorbuto) data del siglo XVIII (1). Con el tiempo llegaron a ser los proveedores y los médicos quienes valoraban la utilidad y la calidad de los medicamentos, instrumentos, equipos, procedimientos o cualquier otra cosa que pudiera denominarse tecnología para atención de la salud. Hasta bien avanzado el siglo XX, sus opiniones se basaban en observación empírica y ha sido solo durante los últimos decenios que se han comenzado a diseñar estudios clínicos rigurosos para fines de evaluación.

En 1931 se creó en los Estados Unidos de América la Administración de Alimentos y Medicamentos (FDA) cuyas tareas en el comienzo consistieron en prevenir los riesgos de enfermar y garantizar la seguridad de los alimentos y de otros productos de uso interno, como los medicamentos, productos biológicos y derivados sanguíneos. Durante los años sesenta los ensayos clínicos se fueron generalizando más y más como requisito para permitir la comercialización de los medicamentos y de algunos otros productos sanitarios. La evaluación de tecnología experimentó un fuerte impulso en la década de los setenta con el establecimiento de la Office of Technology Assessment (OTA) [Oficina de Evaluación de Tecnología], primera dependencia establecida para ese fin en particular. Su primer informe se publicó en 1976 (2).

Teniendo en cuenta que por tecnología se entiende la aplicación del conocimiento empírico y científico a una finalidad práctica, el uso de esta palabra en el campo de la salud empezó refiriéndose a los medicamentos, equipos y dispositivos médicos, procedimientos médicos y quirúrgicos, y modelos de organización y sistemas de apoyo necesarios para atender a los pacientes (3). Esa definición se ha ido expandiendo para abarcar todos los métodos técnicos que se aplican en la atención de las personas (sanas o enfermas) y también para resaltar la importancia de los conocimientos asociados con su uso (4).

Aun más recientemente, el grupo de personas que necesitan saber más acerca de las tecnologías se ha ampliado y diversificado hasta incluir, no solo a los fabricantes y proveedores de servicios, sino a legisladores, funcionarios gubernamentales, administradores de salud, investigadores, gerentes industriales, pacientes y familiares. Además, a la 
información requerida sobre seguridad, riesgo y eficacia, hoy día se han añadido nuevos aspectos como efectividad, factores económicos, consecuencias para la calidad de vida de los usuarios e implicaciones éticas, culturales y sociales de su empleo (5). Es decir, se está pasando de una evaluación principalmente basada en los intereses de los productores a otra que sitúa en primer plano las necesidades individuales y colectivas de los usuarios $(6,7)$.

El presente artículo se ocupa de la tecnología empleada en la atención de salud de las personas para la prevención de riesgos, protección de daños, diagnóstico, tratamiento y rehabilitación y, en especial, la que se emplea en los servicios de salud. Al hablar de su evaluación, nos estamos refiriendo a la forma integral de investigar las consecuencias técnicas - casi siempre clínicas-, económicas y sociales de su empleo tanto a corto como largo plazo, así como sus efectos directos e indirectos, deseados y no deseados. Además, la evaluación de tecnología permite informar a pacientes y clínicos sobre las opciones posibles y proporciona elementos para la toma de decisiones estratégicas relacionadas con la cobertura del seguro de enfermedad o la asignación de recursos, incluida la compra de equipos $(8,9)$. A veces esta evaluación tiende a confundirse con la investigación científica, pero debe recordarse que esta última tiene como objeto producir conocimientos nuevos sobre procesos fisiológicos normales o patológicos. En la investigación aplicada, los resultados procedentes de la investigación básica y de otras fuentes se emplean en busca de soluciones nuevas para problemas de prevención, tratamiento, curación o rehabilitación (10). La evaluación de que hablamos se concibe cada vez más como un proceso de análisis dirigido a estimar el valor y la contribución de cada tecnología sanitaria a la mejora de la salud individual y colectiva, teniendo en cuenta su impacto económico y social (11).

\section{Razones y principios de la evaluación de tecnologías de salud}

Hay varias razones que explican la acentuación reciente del interés en evaluar la tecnología que se emplea en la atención de salud. Una de ellas es la variabilidad de la práctica clínica - por razones que van desde la diversidad epidemiológica local a la accesibilidad y aceptabilidad de métodos y técnicas, las diferencias de capacitación y de conducta del personal y un sinnúmero de posibilidades- que resulta en discrepancias inexplicadas en la frecuencia con que se usan ciertas tecnologías (12-14). Otra razón es el alto grado de incertidumbre sobre el verdadero efecto que tienen muchas intervenciones diagnósticas y terapéuticas de uso común en la salud individual y colectiva y, en particular, en el alivio del sufrimiento, la prolongación de la vida y la mejora de la calidad de la vida (15).

Otra es que el ritmo con que se producen nuevas posibilidades de diagnóstico y terapéutica es muy acelerado, y ello combinado con la presión que ejercen la industria o los posibles usuarios, hace que se introduzcan novedades para uso general mucho antes de que sea posible evaluar rigurosamente sus consecuencias clínicas, éticas, económicas y sociales. Sin embargo, la mayor parte de las nuevas tecnologías tienden a complementar y no a sustituir las anteriores. Ello puede resultar en mayor intervencionismo y deshumanización en la forma de tratar a los pacientes, así como en el aumento de los costos de cada proceso y, en último término, en un incremento del gasto en salud (16).

Estas razones han llevado a la sociedad a interesarse cada vez más en el tema y a los poderes públicos a informarse sobre el grado de utilidad de las tecnologías sanitarias, su uso apropiado, su costo y las condiciones en que deberían o no ser introducidas. Se ha llegado a comprender que no todo lo que es posible desde un punto de vista técnico (es decir, seguro y eficaz) es necesariamente útil (es decir, eficiente y efectivo) para mejorar el estado de salud individual o colectivo. Para controlar los costos sin reducir arbitrariamente el acceso a los cuidados de salud habrá que saber mucho más acerca de la seguridad, efectividad y uso apropiado de los medicamentos, pruebas y procedimientos (15).

Métodos de evaluación. En la evaluación de tecnología se consideran básicamente los siguientes elementos: seguridad, eficacia, efectividad, utilidad, impacto económico, consecuencias sobre la organización de los servicios, implicaciones éticas e impacto social (17-19). Los métodos de evaluación suelen ser tanto generales (aplicables en varias disciplinas de la salud) como específicos (20). Los generales comprenden: 1) analizar y sintetizar la información bibliográfica disponible; 2) aplicar métodos epidemiológicos descriptivos y analíticos, por ejemplo, estudios de cohortes y estudios de casos; 3) realizar ensayos clínicos aleatorios y controlados e investigaciones específicas, y 4) analizar registros y bases de datos. Los métodos específicos consisten en 1) consultar las opiniones de diversos expertos sobre la tecnología de interés; 2) realizar una evaluación socioeconómica, por ejemplo, de costo-eficacia y costo-beneficio, y 3) construir modelos y efectuar simulaciones matemáticas (20-25).

En realidad se utilizan cuantos métodos rigurosos y fuentes de información fiables ayuden a 
producir los conocimientos que se necesitan, ya sean de índole tecnológica, epidemiológica, clínica, sociológica o económica. A veces esa información está disponible y la tarea será recuperarla, ordenarla y analizarla; si no, será preciso obtenerla por medio de un proyecto de investigación.

Ciclo de vida de una tecnología. Puede considerarse que tiene cuatro etapas. En la fase experimental se destacan los investigadores y en ella no suelen actuar los grupos e instituciones que se dedican a evaluar las tecnologías de salud. Los estudios de esta fase son, por lo general, de seguridad y eficacia en sentido estricto y sus resultados son una condición necesaria para pasar a las fases que siguen. Luego, en la fase de implantación se estudian la efectividad, la utilidad clínica, el impacto económico y las consecuencias de la tecnología sobre la organización de los servicios. Ya no es un ámbito experimental sino de ensayo clínico, en condiciones cuidadosamente establecidas desde las perspectivas científica, ética, legal y administrativa. En esta fase suelen comenzar a actuar los organismos y grupos encargados de la evaluación. Sigue la fase de generalización, en la cual se procura establecer el medio en que se aplicará la tecnología, en qué condiciones se ha de difundir y qué mecanismos se emplearán para el seguimiento de sus efectos (esperados y no esperados) a mediano y largo plazo. Esta fase determina cuánto tiempo se ha de seguir utilizando la tecnología y de qué manera, y por lo tanto entra directamente en el campo de acción de los organismos y grupos dedicados a la evaluación. Por último llegará una fase de declive, en la que normalmente se trata de evaluar si la tecnología en cuestión, o una de sus aplicaciones, puede sustituirse ventajosamente por una o varias tecnologías nuevas (18).

Propósitos y destinatarios de la evaluación. En las tecnologías nuevas, la evaluación pretende orientar a los responsables de tomar decisiones sobre tres cuestiones principales: 1) aprobación para el acceso al mercado; 2) aprobación para incluir la tecnología entre las prestaciones financiadas con fondos públicos; $\mathrm{y}$, si procede, 3 ) diseminación adecuada dentro del sistema de salud, especialmente dentro del subsector público.

Con respecto a las tecnologías existentes, las decisiones se refieren a: 1) suspender la financiación pública de tecnologías ya ineficientes; 2) generalizar nuevas aplicaciones de tecnologías en uso en el sistema de salud público; 3) retirar la tecnología del mercado o suprimir una de sus indicaciones (por ejemplo, debido a efectos secundarios indeseables, como fue el caso de la talidomida) (26).
Si bien las autoridades sanitarias no suelen habitualmente retirar del mercado procedimientos diagnósticos o terapéuticos solo porque sean ineficientes, los pueden convertir en obsoletos dando publicidad a su ineficiencia. Un buen ejemplo de ello es la reducción de histerectomías en lugares donde se han difundido extensamente indicaciones más restrictivas (27). Cuando cuentan con evaluaciones precisas, las autoridades pueden también retirar la financiación pública de tecnologías obsoletas o no suficientemente probadas, en beneficio de otras que reúnan los requisitos adecuados. Inversamente, hay numerosos ejemplos de tecnologías costo-efectivas que actualmente están subutilizadas (28).

En un sistema de salud, los resultados de la evaluación afectan sobre todo a los ámbitos de regulación y provisión de las tecnologías. En cuanto a regulación, las autoridades tienen que tomar decisiones que afectan al registro, la autorización y la aprobación de las condiciones básicas de uso (fundamentalmente, de los medicamentos y los productos sanitarios); a la inclusión o no de la tecnología en la cobertura del seguro de salud público, y a las condiciones concretas de su financiamiento. Estas decisiones dependen de la información obtenida de evaluaciones apropiadas.

En lo que se refiere a provisión, los profesionales de salud que usan una tecnología tienen que saber con todo detalle en qué condiciones es segura, eficaz, eficiente y aceptable, y las ventajas e inconvenientes de distintas opciones (29). Los pacientes y sus familiares deben ser informados sobre muchos de los aspectos anteriores para que puedan hacer una elección siempre que sea posible. Por su parte, los gerentes y administradores desean conocer los problemas y adaptaciones que entraña la implantación de una tecnología, cuánto tiempo de utilidad puede esperarse, las condiciones de recuperación del costo de la inversión y posibles aplicaciones adicionales. En cuanto a la industria, le incumbe saber los resultados de la difusión de las tecnologías, ya que son una fuente de información relevante para el diseño de nuevas tecnologías o de nuevas aplicaciones de las que ya están en uso.

Fuera de los sistemas de salud, los medios de comunicación y el público en general muestran cada vez más interés en conocer los resultados de las evaluaciones de tecnologías, por muchas de las razones arriba expuestas. Ese interés se destaca especialmente cuando la evaluación influye en las decisiones de las autoridades de salud por su fuerte impacto ético, de salud pública o económico.

Evaluaciones de nivel nacional e internacional. Las tecnologías para la atención de salud son bienes 
y servicios que, en su mayor parte, han sido concebidos para un mercado mundial. En los países industrializados, el proceso que va desde el desarrollo hasta el uso de las tecnologías se realiza tanto a nivel internacional como nacional. En el contexto nacional, ese proceso varía de acuerdo con las características del sistema de salud y a veces de las políticas científicas y comerciales de cada país. Los países que tienen sistemas de salud de financiación mayormente pública como Canadá, España, Noruega, Reino Unido o Suecia, han estado creando organismos dedicados a la evaluación de tecnologías. Su actividad comenzó centrándose en las más costosas - por ejemplo, los equipos de imagen, de laboratorios y de cirugía-, pero poco a poco ha abarcado el resto de las tecnologías. Un logro importante de esta tendencia ha sido convertir la evaluación de tecnologías empleadas en los servicios de salud en un componente esencial de las políticas que pretenden conciliar la calidad con la contención de costos. En algunos países la evaluación está a cargo de instituciones académicas y organismos privados. En casi todos se percibe cierta dispersión de actividades y se siente la necesidad de coordinar más a los distintos grupos de evaluadores $(30,31)$.

En 1989, Canadá creó la Oficina Canadiense de Coordinación para la Evaluación de Tecnología de la Salud (CCOHTA), una corporación pública sin ánimo de lucro gobernada por un consejo de 13 representantes de las provincias y territorios. Ese consejo trabaja con dos comités consultivos, uno sobre medicamentos y el otro sobre el resto de las tecnologías. Además existen el Consejo Coordinador de Evaluación de Tecnologías de la Salud, dependiente del gobierno provincial de Quebec, y la Alberta Heritage Foundation.

En los Estados Unidos, la Office of Technology Assessment fue suprimida en 1995, pero se ha mantenido la FDA y han proliferado las empresas privadas (con y sin ánimo de lucro) dedicadas a la evaluación de tecnología. Se ha fortalecido el papel que desempeñan las sociedades científicas y profesionales, y el enfoque se ha ido desplazando del nivel general de regular la introducción al más concreto de controlar su utilización.

A nivel internacional, desde 1985 existe la International Society of Technology Assessment of Health Care (ISTAHC) que es una sociedad científica de libre afiliación con unos 1000 miembros individuales y corporativos de más de 30 países, que representan universidades, industrias, compañías de seguros, instituciones de salud, profesiones de salud y gobiernos. La ISTAHC promueve actividades internacionales, entre ellas una reunión internacional anual, y publica una revista cuatrimestral de referencia obligada (32). Recientemente se consti- tuyó en su seno un grupo de interés para el desarrollo de la evaluación de tecnología de salud en los países en desarrollo y, por iniciativa de la OPS, su XIII Reunión Anual en 1997 incluyó un panel sobre el tema de la reforma de los sistemas de salud y la evaluación de tecnologías en América Latina y el Caribe.

Otros organismos internacionales son la International Network of Agencies for Health Technology Assessment (INAHTA) establecida en 1993; el programa EUR-ASSESS de 1994 en la Unión Europea; y la red de centros denominada "Colaboración Cochrane", en honor del ilustre epidemiólogo clínico Archie Cochrane (33-35).

\section{La OMS y la evaluación de tecnologías para la salud}

El interés de la OMS por este tema data de la famosa Declaración de Alma-Ata en 1978, en la cual la atención primaria de salud se definió como "la asistencia sanitaria esencial basada en métodos y tecnologías prácticos, científicamente fundados y socialmente aceptables, puesta al alcance de todos los individuos y familias de la comunidad ..." (36). En el informe de la Conferencia, sección de Recomendaciones, apartado 12, se "recomienda que los gobiernos, las instituciones de investigación y de enseñanza, las organizaciones no gubernamentales $\mathrm{y}$, sobre todo, las comunidades, desarrollen ... tecnologías y métodos aptos para mejorar la salud, científicamente válidos, adaptados a las necesidades locales, aceptables para la comunidad, y mantenidos por la propia población ... a un coste abordable para la comunidad y para el país" (36). En la Serie de Informes Técnicos de la OMS se incluyen con frecuencia creciente informes sobre la elección apropiada, uso racional y control de calidad de diversas tecnologías promocionales, preventivas, diagnósticas y terapéuticas.

En 1984, la Oficina Regional de la OMS para Europa exhortó a todos los Estados Miembros a que establecieran un mecanismo formal para la valoración sistemática de las tecnologías de salud antes de 1990. A mediados de los noventa, ese objetivo se había cumplido solo en la mayoría de los países de Europa Occidental.

Desde 1991, la OMS ha realizado reuniones de expertos sobre evaluación de tecnología y, para promover esa actividad en los países en desarrollo, reunió a un grupo de trabajo que formuló sus propuestas en 1994. Además, en el presupuesto bienal de la OMS para 1996-1997 hay un capítulo dedicado a calidad y tecnologías sanitarias, donde se resumen las actividades realizadas en estas áreas en el bienio precedente. 


\section{Actividades de evaluación en América Latina y el Caribe}

Como en la mayoría de los países en desarrollo, en América Latina y el Caribe el desarrollo de tecnología se ha basado fundamentalmente en la transferencia de tecnologías elaboradas en los países desarrollados. En muchos casos, la transferencia no ha sido apropiada, al no ir acompañada de adaptaciones a las circunstancias económicas, sociales y culturales de los países receptores. A comienzos de los años ochenta se identificaron como problemas en esos países la falta de tecnologías básicas, la utilización indiscriminada de tecnología costosa, la deficiencia de las políticas y normas que regulan la introducción y el uso de tecnologías, y el subdesarrollo de las tecnologías de apoyo (de los sistemas de información gerencial en particular) así como las desigualdades de acceso a las disponibles (37).

A comienzos de la década de 1990, la situación dejaba mucho que desear. Incluso en países con grados de desarrollo medianos o más altos, llamaba la atención la falta de información confiable sobre los mecanismos de introducción, distribución y uso de las tecnologías de salud más comunes. En segundo lugar era evidente que, como resultado de la crisis de la década precedente, los países no habían destinado fondos adecuados al mantenimiento y la reposición de instalaciones y equipos. En los hospitales públicos de Centroamérica y Panamá, los edificios tenían en promedio entre 30 y 35 años y cerca de la mitad de los equipos o no funcionaban o lo hacían deficientemente. Los países dedicaban menos de 3,5\% y, a menudo, menos de $1 \%$ del presupuesto corriente a conservación y mantenimiento, y el personal para esas tareas era escaso y mal calificado para el trabajo (38).

Por otra parte, algunos países continuaban recibiendo donaciones de equipo que no correspondía a necesidades expresadas, no era acorde con las regulaciones nacionales, y para el que no había situaciones de manejo adecuadas, ni personal adiestrado, ni suficientes recursos para mantenimiento (39). La disponibilidad y utilización de equipos de diagnóstico por imágenes, radioterapia, laboratorios y bancos de sangre era muy variable. En los países grandes, con altos índices de urbanización, había servicios muy avanzados, casi siempre localizados en los hospitales de las grandes capitales, especialmente si eran privados. En estos, los indicadores de disponibilidad, uso y calidad eran similares a los de los países industrializados. Fuera de esas ciudades, o en países de menor desarrollo, la situación era sustancialmente peor. Solo dos de los siete servicios de diagnóstico por imagen visitados en un país contaban con dosimetría personal y en cuatro de ellos no había sistema de control de la calidad.
En materia de medicamentos, se observaba un proceso en dos direcciones. Por un lado, como consecuencia de la tendencia a la globalización e integración subregional, se propendía a desregular las condiciones de registro y los precios y, por otro, a fomentar el uso racional y mejorar la calidad de la atención farmacoterapéutica. Según una encuesta de 13 países, solo la mitad de ellos contaban con organismos que actuaran como comisiones nacionales de medicamentos y los que existían no tenían la capacidad de gestión. Solo en una cuarta parte de los países se habían adoptado oficialmente las normas de buenas prácticas de fabricación. En pocos casos se había logrado aumentar la disponibilidad de medicamentos genéricos en el mercado. Se observó también la tendencia a vender medicamentos sin receta o en establecimientos comerciales que no cuentan con farmacéutico. En el sector público, había disminuido el número de países con sistemas centralizados de compra, se había debilitado la capacidad gerencial del sector público y había aumentado el número de instituciones que compran medicamentos no incluidos en las listas básicas y el de países que habían establecido diversas modalidades de aportación del usuario. La información recibida por los médicos provenía casi exclusivamente de la industria farmacéutica. Por último, se habían publicado muy pocas evaluaciones sobre tecnología (39).

En América Latina y el Caribe hay una enorme carencia de dispositivos médicos y material hospitalario. En 1993, los países de esas zonas representaban un mercado de productos de salud de alrededor de US\$ 16000 millones. Ese año Brasil invirtió $\$ 7,4$ per cápita en esos productos y México, \$5,5, mientras que Canadá destinó \$82, Japón \$94, Alemania $\$ 108$ y Estados Unidos \$140. Pese a la falta de estudios que lo comprueben, hay razones para pensar que la distribución interna de los productos médicos, especialmente los de tecnología más moderna, se distribuyen muchas veces en relación inversa a las necesidades de salud (39).

La transición demográfica por el aumento de la esperanza de vida y predominio de las enfermedades crónicas y accidentes como causas de mortalidad y morbilidad, junto con las mejoras económicas en muchos países están presionando al alza del gasto en tecnología durante los próximos años. La evaluación tendrá un papel muy importante para orientar el gasto hacia los materiales y equipo más apropiados y costo-efectivos.

Actitud hacia la evaluación de la tecnología. A pesar de que la mayor parte de los países de América Latina y el Caribe están llevando a cabo reformas de sus sistemas de salud, ha habido poco inte- 
rés en considerar las tecnologías de salud en función de los gastos que representan y su evaluación para mayor efectividad y eficiencia. En la Cumbre de las Américas de 1994 se reafirmó el interés de los gobiernos en promover reformas para garantizar el acceso equitativo a los servicios básicos de salud, pero allí tampoco fueron mencionados los problemas que plantean el costo y la escasez de tecnologías y la necesidad de evaluarlas. Por cierto, la Cumbre encargó a la OPS, el Banco Interamericano de Desarrollo y el Banco Mundial que organizaran una reunión especial sobre las reformas de salud en las Américas y cada país preparó un informe sobre las estrategias e iniciativas en curso $(40,41)$. Aparte de los medicamentos, mencionados por seis de 37 países, ninguno mencionó la evaluación de tecnologías como prioridad. Además, en una encuesta realizada entre 1994 y 1995 se identificaron 103 organizaciones dedicadas a dicha evaluación en 24 países. Solo una estaba en un país de América Latina o el Caribe (42).

Esa indiferencia podría deberse a que se trata de un campo relativamente nuevo y, de los organismos y agencias dedicados a esas funciones, ninguno está establecido en las zonas geográficas que nos ocupan. Desde una perspectiva técnica, la evaluación de tecnología es una tarea compleja, que involucra habilidades y conocimientos diversos: investigación básica y aplicada, enfermería, epidemiología, ingeniería, economía, gestión y otras. También debe tenerse en cuenta que en un contexto de liberalización económica y globalización, la evaluación de tecnología puede a veces ser percibida como una nueva forma de intervencionismo estatal o de burocracia.

Sin embargo, no puede decirse que la evaluación de tecnología no esté presente en la actividad diaria de las autoridades de salud, clínicos y usuarios. La globalización económica y el acceso electrónico a la información tienden a diseminar con gran rapidez las noticias de avances tecnológicos y los resultados de su evaluación. Además, ciertos organismos internacionales están destacando la importancia de evaluar los dispositivos como una forma de mejorar la calidad, efectividad y asignación de recursos, lo cual contribuye a reducir las inequidades en salud (43-45). Otras razones son que la relación entre incentivos profesionales y el uso de la tecnología se empieza a ver claramente en algunos países; los usuarios han ido adquiriendo mayor conciencia de las implicaciones éticas y legales de muchas tecnologías; y que una de las estrategias de reforma del sector es el establecimiento de paquetes o conjuntos básicos de intervenciones costo-efectivas, las cuales exigen ejercicios más o menos complejos de evaluación (46).

\section{La evaluación de tecnología y la OPS}

El concepto de evaluación de la tecnología se manifestó en la OPS hace mucho tiempo pues, junto con la estrategia de salud para todos en 1978, se difundió la idea de tecnologías apropiadas. En 1983 se estableció la Unidad de Desarrollo de Tecnologías en Salud, que impulsó una activa política de publicaciones, varios estudios en colaboración, reuniones de grupos de consulta internacionales y seminarios $(47,48)$. En las orientaciones estratégicas y prioridades programáticas (OEPPS) trazadas por la OPS para el cuadrienio de 1991 a 1994 y aprobadas en 1990, el desarrollo tecnológico figuraba como una de las prioridades para el fortalecimiento de los servicios de salud. En las OEPPS para el período de 1995-1998, se expresa preocupación por la aparición de nuevas tecnologías, su incorporación al campo de la salud y el acceso a información sobre ellas. Se busca, como líneas de acción concretas, promover la cooperación entre los países para el desarrollo y uso de la tecnología, apoyar la formulación de políticas sobre medicamentos esenciales y fortalecer el desarrollo de servicios de laboratorio (43).

Últimamente, la importancia que ha adquirido este rubro se refleja en su frecuente mención en los diferentes documentos de la Organización. En el informe del Director aparece regularmente un resumen de las actividades que se han realizado en tecnología y medicamentos esenciales (49). En un documento reciente se señala que la acción de la OPS se orientará, entre otras cosas, al "fortalecimiento de la capacidad de [. . .] evaluación de tecnologías, medicamentos y otros insumos considerados críticos" (50).

La tecnología de salud y la reforma de los sistemas en América Latina y el Caribe. Fortalecer la evaluación de tecnología en salud se considera un componente indispensable para la reforma del sector y el desarrollo de los servicios. A continuación se plantean ideas que se espera sean de interés para todas las personas involucradas en esas tareas.

¿Dónde empezar? En América Latina y el Caribe, la frase tecnologías de salud suele traer a la memoria equipos complejos y costosos. Si se habla de evaluación de tecnologías, se piensa en su evaluación antes de registrarlas y comercializarlas. Sin embargo, los hospitales de tercer nivel de algunos países ya cuentan con guías de práctica clínica que usan ocasionalmente médicos y enfermeras.

La elaboración y difusión de guías nacionales (o subnacionales, si procede) de práctica clínica 
para el manejo adecuado de situaciones clínicas de gran impacto, por ejemplo, para el tratamiento de la úlcera gastroduodenal ha sido frecuentemente el primer paso en evaluación de tecnologías. En el proceso de elaboración es necesario discutir en profundidad las tecnologías y la necesidad de establecer prioridades en la evaluación (51). La experiencia con los medicamentos puede ser útil como analogía. Los conceptos de seguridad, eficacia, efectos no deseados y costo-efectividad son más o menos idénticos así como muchos elementos de la filosofía básica y de la metodología de trabajo.

\section{Equidad, calidad y efectividad frente a contención} de costos. Durante los últimos 20 años se han publicado varios informes en los que se señala que en los Estados Unidos y otros países desarrollados se invierte una proporción considerable de los recursos de salud en procedimientos clínicos inútiles, perjudiciales o dudosos (52). La eliminación de esos tipos de procedimientos y de las indicaciones inapropiadas podría mejorar la calidad y la efectividad y reorientar el gasto sanitario para que sea más costo-efectivo. Puede ser una estrategia menos compleja y más aceptable que definir paquetes o conjuntos básicos de intervenciones costo-efectivas.

Vinculación de los profesionales de salud con la evaluación. El progreso acelerado de las tecnologías y de la forma de tratar a los pacientes ha contribuido a variar poderosamente las modalidades de atención y la distribución de funciones de distintos niveles. Muchos procedimientos antes restringidos al nivel hospitalario pueden ahora realizarse en el nivel primario o aun en el domicilio del paciente. Todo ello fortalece el papel de médicos y enfermeras como consejeros de los pacientes y sus familiares y también de los gerentes y encargados de tomar decisiones políticas. Estos rara vez pueden tomar decisiones con respecto a nuevas construcciones, adquisición o reemplazo de equipo, aprobación de nuevas aplicaciones, etc., sin consultar con grupos representativos de especialistas. Incluso en sitios carentes de recursos, cuando se trata de preparar proyectos para buscar financiación externa, se necesita ese tipo de evaluación. Esta situación, que se llamó hace algunos años "la tercera revolución en medicina", se caracteriza por la necesidad de dotarse de procedimientos y recursos para desarrollar una evaluación crítica permanente de las tecnologías empleadas en los servicios de salud. Es una situación que plantea estimulantes retos científicos y una gran responsabilidad profesional (53).
La evaluación como componente del papel regulador de los ministerios de salud. El fortalecimiento del papel rector de los ministerios es una de las estrategias para la reforma sectorial y la evaluación de tecnologías debería ser un componente fundamental de esa estrategia. Las entidades que ejecuten ese trabajo pueden ser agencias independientes, públicas o vinculadas con universidades y otras instituciones; es decir, variarán de un país a otro. Actualmente en los Estados Unidos no hay un solo organismo público especializado en evaluación de tecnologías. La OTA, que ya no existe, dependía del Congreso, la FDA y la Agency for Health Care Policy Research son parte del Departamento de Salud y Asistencia; el Instituto de Medicina forma parte de la Academia Nacional del Ciencias y ECRI es una organización privada independiente sin ánimo de lucro.

Los países de América Latina y el Caribe podrían empezar estableciendo pequeñas unidades de evaluación en los ministerios de salud, las cuales podrían a su vez integrar la secretaría de un comité nacional que incluyera representantes de universidades, institutos de investigación, sociedades científicas y otras organizaciones públicas y privadas. Este comité establecería las prioridades y el plan de trabajo así como los recursos necesarios y la secretaría trabajaría en estrecho contacto con una red de sociedades científicas y grupos profesionales para llevar a cabo las evaluaciones incluidas en el plan.

En los pocos países que cuentan con organismos reguladores autónomos, deberá plantearse si la unidad antes mencionada se inscribe en ellos, depende de la autoridad de salud o es una agencia independiente. Esta última variedad tiene la ventaja de poder recibir financiación tanto del sector público como del privado. En cualquier caso, el grupo inicial debe ser pequeño, estable, multidisciplinario, independiente y muy bien cualificado, y capaz de trabajar muy de cerca con los grupos y las instituciones sanitarias de mejor nivel del país. Puesto que la evaluación de tecnología consume tiempo y dinero, es esencial organizar adecuadamente los recursos para lograr los objetivos de las unidades evaluadoras.

La cooperación técnica internacional. Tres razones fundamentales para estimular la cooperación entre países en la evaluación de tecnologías son evitar la duplicación de estudios en distintos países, facilitar el intercambio eficiente y oportuno de información y experiencia, y progresar en el desarrollo de nuevos conocimientos para mejorar el funcionamiento de los sistemas de salud. 
La cooperación entre los países de América Latina y el Caribe y los Estados Unidos, Canadá y las naciones europeas permitiría diseñar estrategias propias después de considerar los resultados de programas llevados a cabo en los países con más experiencia en este campo. Algunos países europeos incluyen la evaluación de tecnologías entre las áreas de cooperación con países en desarrollo. También el Organismo Canadiense para el Desarrollo Internacional (CIDA) apoya los esfuerzos para introducir tecnologías apropiadas.

En cuanto a cooperación entre los propios países de América Latina y el Caribe, la experiencia con los medicamentos puede servir de orientación. En mayo de 1994, los gobiernos de los países andinos suscribieron un Plan Cuadrienal (1994-1997) sobre Estrategias, Oferta, Regulación, Calidad y Uso Racional de Medicamentos. Además, en junio del mismo año, los presidentes centroamericanos decidieron crear un mercado libre para 40 medicamentos esenciales y evaluar conjuntamente el funcionamiento de programas sobre indicadores de uso, y regulación de las prescripciones y dispensación. Por otra parte, en abril de 1996, la Reunión de Ministros de Salud de MERCOSUR (Argentina, Brasil, Paraguay y Uruguay) se organizó en torno a los dos temas de calidad y tecnología de salud. Si bien la reunión no tuvo resultados prácticos, sirve para señalar una vía de aproximación subregional al tema.

Más importante aún, recientemente las autoridades regulatorias de los países de cuatro subregiones (Norteamérica, Centroamérica, Área Andina y MERCOSUR) han comenzado los trabajos para armonizar los requisitos y procedimientos para el registro de medicamentos. En el Área Andina se han empezado a elaborar las Normas Farmacológicas Andinas para los productos comunes de la subregión. En Centroamérica se está iniciando un proyecto similar.

Función de la OPS. La OPS puede servir como facilitadora prestando apoyo a los países en el establecimiento de políticas y mecanismos para impulsar la evaluación de tecnologías de salud. Ese apoyo puede expresarse en las siguientes acciones:

1. Analizar la situación y las necesidades.

2. Establecer prioridades.

3. Identificar grupos e instituciones nacionales relevantes en este campo.

4. Impulsar la organización de unidades y organismos coordinadores.

5. Facilitar la conexión con organismos, grupos y redes internacionales.
6. Organizar talleres y seminarios sobre metodología y práctica de la evaluación de tecnología.

7. Prestar cooperación técnica en materia de regulación, difusión y evaluación del uso de algunas tecnologías en particular.

8. Participar en conferencias internacionales sobre la evaluación de tecnologías relevantes.

9. Establecer y reforzar los mecanismos de diseminación de resultados e intercambio de experiencias.

10. Evaluar el impacto de las recomendaciones presentadas en los informes.

La experiencia acumulada —en asociación con la OMS - en programas como los de medicamentos y vacunas, imaginería y radioterapia, radioprotección, laboratorios clínicos y bancos de sangre constituye una buena base para seguir avanzando. Además, se cuenta con los centros colaboradores de la OMS en la Región, con los cuales existen relaciones que se intensificarán en el futuro.

En la actualidad, las dos limitaciones principales en la Región son, por un lado, la falta de comprensión de la importancia de la evaluación tecnológica para lograr los objetivos de reforma de los sistemas y servicios de salud y su relación con el papel rector de los ministerios de salud y, por otro lado, la falta de un número mínimo de personal formado en la metodología y práctica de la evaluación de tecnologías, estable y dedicado, y con suficientes conexiones nacionales e internacionales. Dadas esas limitaciones, la OPS ha comenzado a cooperar con dos líneas de trabajo relevantes. Una es la organización con recursos propios de tres talleres sobre evaluación de tecnologías, uno nacional en Cuba y otros dos subregionales en México y Chile. Se está planificando otro más para 1997 en Bogotá, Colombia. En otra línea de trabajo, la OPS está sirviendo de intermediario desinteresado entre las autoridades sanitarias (y grupos y unidades de los países) y otras iniciativas de cooperación internacional tales como ISTAHC e INAHTA.

\section{SYNOPSIS}

\section{Assessment of health care technology}

This report explains how and why to evaluate the technology employed in health care. This technology includes not only medicines, instruments, equipment, and procedures used directly on patients, but also the organizational models and support systems that make health care possible. During 
the various stages of a technology's life cycle, an assessment is made of several different characteristics, such as safety and effectiveness, clinical utility, economic impact, and effect on the organiztion and provision of services. Originally, the ways of assessing and testing technical methods were only of interest to manufacturers and service providers. Today, however, the interested parties include legislators, government officials, health administrators, researchers, industrial managers, and, certainly, patients and their families. Therefore, health technology assessment is a highly important activity and its promotion is an element of PAHO's technical cooperation with the Member States.

\section{REFERENCIAS}

1. Lind J. Una investigación sobre la naturaleza, las causas y la curación del escorbuto. En: Buck C, Llopis A, Nájera E, Terris M. El desafío de la epidemiología: problemas y lecturas seleccionadas. Washington, DC: Organización Panamericana de la Salud; 1988: 20-24. (Publicación científica 505).

2. United States, Office of Technology Assessment. Development of medical technologies: opportunities for assessment. Washington, DC: US Government Printing Office; 1976.

3. United States, Office of Technology Assessment. Assessing the efficacy and safety of medical technologies. Washington, DC: US Government Printing Office; 1978.

4. World Health Organization. Technology for health in the future: a report of the working group (draft). Geneva: WHO; May 1997.

5. Menon D, Marshall D. The internationalization of health technology assessment. Int I Technol Assess Health Care 1996;12(1):45-51.

6. Tugwell $\mathrm{P}$, Sitthi-Amon $\mathrm{C}, \mathrm{O}^{\prime}$ Connor A, Haatcher-Roberts J, Bergevin Y, Wolfson M. Technology assessment: old, new, and needs-based. Int J Technol Assess Health Care 1995;11(4):650-652.

7. Ong BN. The lay perspective in health technology assessment. Int J Technol Assess Health Care 1996;12(3):511-517.

8. Institute of Medicine. Setting priorities for health technology assessment: a model process, En: Field MJ, ed. Setting priorities for clinical practice guidelines. Washington, DC: National Academy Press; 1995: 77-110.

9. United States, Office of Technology Assessment. Identifying health technologies that work: searching for evidence. Washington, DC: US Government Printing Office; 1994.

10. Banta DH. Health care technology as a policy issue. Health Policy 1994;30:1-20.

11. Granados A. La evaluación de las tecnologías médicas. Med Clin (Barcelona) 1995;104:581-588.

12. Wennberg JE. Dealing with medical practice variations: a proposal for actions. Health Aff 1984;3:6-32.
13. Chassin MR, Brook RH, Park RE, Keesey J, Fink A, Kosecoff J, et al. Variations in the use of medical and surgical services by the Medicare population. $N$ Engl J Med 1986;314:285-290.

14. Conde J. Evaluación de las tecnologías sanitarias y su relación con la calidad asistencial. En: La formación de los profesionales de la salud: escenarios y factores determinantes. Madrid: Fundación BBV; 1996.

15. Relman AS. Assessment and accountability: the third revolution in medical care. N Eng J Med 1988;319:1220-1222.

16. Blanco A, De Bustos A. El gasto sanitario público en España: diez años de Sistema Nacional de Salud. En: XVI Jornadas de Economía de la Salud, Valladolid, junio, 1996.

17. Bautista RN, Banta HD, Jonsson E, Hodge M, Gelband H. Lessons from the eight countries. Health Policy 1994;30: 397-421.

18. Banta HD, Luce BR. Health care technology and its assessment. Oxford: Oxford University Press; 1993.

19. Lázaro y de Mercado P. Evaluación de tecnología médica. Madrid: MCQ Ediciones; 1994. (Papeles de Gestión Sanitaria).

20. Ministerio de Sanidad y Consumo, Instituto de Salud Carlos III, Agencia Española de Evaluación de Tecnologías Sanitarias (AETS). Evaluación epidemiológica de tecnologías de salud. Madrid: AETSInstituto de Salud Carlos III; 1996.

21. US Preventive Task Force. Guide to clinical preventive services. Baltimore, Maryland: Williams and Wilkins; 1996.

22. Jovell AJ, Navarro-Rubio MD. Evaluación de la evidencia científica. Med Clin (Barc) 1995;105:740-743.

23. Peiró S, Portella E. No todo es acuerdo en el consenso: limitaciones de los métodos de consenso en los servicios de salud. Gac Sanit (Barc) 1993;7:294-300.

24. Briones E, Marín I, Álvarez R, Reyes A. Fundamentos de consenso en el ámbito de las ciencias de la salud. En: Berra A, Marín I, Álvarez R, eds. Metodología de expertos: consenso en medicina. Granada: EASP; 1996:13-24.

25. Davidoff AJ, Powe NR. The role of perspective in defining economic measures for the evaluation of medical tecnology. Int J Technol Assess Health Care 1996; 12(1):9-21.

26. Conde J, Infante A. La ordenación de prestaciones del Sistema Nacional de Salud. Med Clin (Barc) 1995;104:503-505.

27. The Minister of Health of the Netherlands. Medical technology assessment and efficiency in health care. Rijswijk: Ministry of Health, Welfare and Sport; 1996.

28. Bloom BS, Frendrick AM. The tension between cost containment and the under-utilization of effective health services. Int J Technol Assess Health Care 1966;12(1):1-8.

29. Pelletier D, Duffeld C, Adams A, Crisp J, Nagy S, Murphy J. The impact of the technological care environment on the nursing role. Int J Technol Assess Health Care 1996;112(2):358-366.

30. Sassi F. Health technology assessment: an introduction. Eurohealth 1996;2(4): 9-10.

31. Banta HD. Coordination of health technology assessment in Europe. Eurohealth 1996;2(4):11-13.

32. Battista R, Tombs G. International growth of health technology assessment. Eurohealth 1996;2(4):14-15.

33. Hailley D, Sampietro-Colom L, Marshall D, Rico R, Granados A, Asua J et al. Efectividad de la medida de densidad ósea y de los tratamientos asociados en la prevención de fracturas. INAHTA Newwletter 1996:sept.

34. Banta HD, coord. Report from the EURASSESS Project. Int $J$ Technol Assess Health Care 1997;13(2):133-143.

35. Cochrane Collaboration. Cochrane Collaboration handbook. Oxford: Oxford University Press; 1994.

36. Organización Mundial de la SaludUNICEF. Alma-Ata 1978: atención primaria de salud: informe de la Conferencia Internacional sobre Atención Primaria de Salud, Alma-Ata, URSS, 6-12 de septiembre de 1978. Ginebra:OMS;1978.

37. Lamm RD. The ethics of excess. Public Health Rep 1996;3:218-223.

38. Organización Panamericana de la Salud. Agenda de propuestas de evaluación de tecnologías en programas de salud del 
adulto: reunión de consulta. Washington, DC; 1985. (Documento PNSP/85/ 04/11).

39. Organización Panamericana de la Salud. Establecimientos hospitalarios; Medicamentos e inmunobiológicos. En: Vol 1 Las condiciones de salud en las Américas. Edición de 1994. Washington, DC: OPS; 1994: 353-360; 371-380. (Publicación científica 549).

40. Summit of the Americas. Plan of action (paragraph 17). Miami, December 9-11, 1995.

41. Pan American Health Organization. Progress of activities in health sector reform. $26^{\text {th }}$ Meeting of the Subcommittee on Planning and Programming, Washington, DC, 25-27 March 1996. (Documento SPP26/7[orig. Spanish]).

42. Perry S, Gardner E, Thamer M. The status of health technology assessment worldwide: results of an international survey. Int J Technol Assess Health Care 1997;13(1):81-98.

43. Organización Panamericana de la Salud. Orientaciones estratégicas y programáticas. Washington, DC: OPS;1995.

44. BID, BIRF, CEPAL, OEA, OPS, UNFPA UNICEF, USAID. Acceso equitativo a servicios básicos de salud: hacia una agenda regional para la reforma del sector salud. Washington, DC: septiembre de 1995. (Documento fotocopiado)

45. World Bank. World Development Report 1993: investing in health. New York: Oxford University Press; 1993:135-155.

46. Colombia, Ministerio de Salud. Tm 1, La reforma de la seguridad social en salud. Bogotá: Ministerio de Salud; 1994.

47. Panerai RB, Peña Mohr J. Health technology assessment: methodologies for developing countries. Washington, DC: Pan American Health Organization; 1989.
48. Organización Panamericana de la Salud. Pro salute novi mundi: historia de la Organización Panamericana de la Sa-lud. Washington, DC: OPS; 1992:161; 242-249.

49. Organización Panamericana de la Salud. Tecnología y medicamentos esenciales. En: En busca de la equidad: informe anual del director, 1995. Washington, DC: OPS, 1995:30-32. (Documento oficial 277).

50. Organización Panamericana de la Salud. La cooperación de la OPS ante los procesos de reforma sectorial. Washington, DC; marzo de 1997. (ISBN 92750734 0).

51. Field MJ, ed. Setting priorities for clinical practice guidelines Washington, DC: National Academy Press; 1995.

52. Brook RH. Testimony before the Committee on Ways and Means. Washington, DC: US House of Representatives, April 16, 1991.

53. Blumenthal D. Quality of care: what is it? N Engl J Med 1996;335:891-893. 